\title{
Congestion management with demand response considering uncertainties of distributed generation outputs and market prices
}

\author{
Linna $\mathrm{NI}^{1}$, Fushuan $\mathrm{WEN}^{2}$, Weijia LIU ${ }^{1}$, Jinling MENG ${ }^{3}$, \\ Guoying LIN $^{3}$, Sanlei DANG ${ }^{3}$

\author{
Guring LIN
} \\ (1)
}

Abstract In recent years, much attention has been devoted to the development and applications of smart grid technologies, with special emphasis on flexible resources such as distributed generations (DGs), energy storages, active loads, and electric vehicles (EVs). Demand response (DR) is expected to be an effective means for accommodating the integration of renewable energy generations and mitigating their power output fluctuations. Despite their potential contributions to power system secure and economic operation, uncoordinated operations of these flexible resources may result in unexpected congestions in the distribution

CrossCheck date: 24 November 2016

Received: 6 August 2016/Accepted: 24 November 2016/Published online: 30 December 2016

(c) The Author(s) 2016. This article is published with open access at Springerlink.com

$\triangle$ Fushuan WEN

fushuan.wen@gmail.com

Linna NI

nilinna918@163.com

Weijia LIU

liuweijiamarcel@gmail.com

Jinling MENG

jinling_meng@163.com

Guoying LIN

13500015251@139.com

Sanlei DANG

13570449055@139.com

1 School of Electrical Engineering, Zhejiang University, Hangzhou 310027, Zhejiang, China

2 Department of Electrical and Electronic Engineering, Universiti Teknologi Brunei, Bandar Seri Begawan BE1410, Brunei

3 Electric Power Research Institute of Guangdong Power Grid Co. Ltd., Guangzhou 510600, Guangdong, China

system concerned. In addition, the behaviors and impacts of flexible resources are normally highly uncertain and complex in deregulated electricity market environments. In this context, this paper aims to propose a DR based congestion management strategy for smart distribution systems. The general framework and procedures for distribution congestion management is first presented. A bi-level optimization model for the day-ahead congestion management based on the proposed framework is established. Subsequently, the robust optimization approach is introduced to alleviate negative impacts introduced by the uncertainties of DG power outputs and market prices. The economic efficiency and robustness of the proposed congestion management strategy is demonstrated by an actual $0.4 \mathrm{kV}$ distribution system in Denmark.

Keywords Distribution system, Congestion management, Demand response, Load aggregators, Uncertainty, Robust optimization, Bi-level linear programming

\section{Introduction}

The past decade has witnessed rapid development and implementation of smart grid technologies in modern power systems. Specific to distribution systems, the integration of distributed energy resources (DERs) such as distributed generations (DGs) [1], energy storage devices [2, 3], active loads [4-11] and electric vehicles (EVs) [10-12], has greatly changed the operation conditions of the distribution system concerned. DERs are believed to play critical roles in shifting peak loads [7], improving power qualities, enhancing operation efficiency [13] and mitigating possible congestions at distribution system levels [14] in future power systems. On the other hand, the 
increasing penetration of renewable energy sources as well as the popularization of plug-in EVs will introduce significant uncertainties, and impose challenges to the optimal operation of distribution systems. As a result, efficient and reasonable utilization of integrated DERs, especially in the electricity market environment, will become a more and more important issue for distribution system operators (DSOs) in the near future.

Among all negative impacts that DERs may introduce to the distribution system concerned, congestion is likely to be the most fatal one as it may directly damage the devices such as distribution transformers and feeders. In general, congestions in a distribution system may be caused by various factors, e.g. very high power consumption during peak hours, concentrated charging of EVs and excessive power generation from DGs. Distribution system congestion has been studied in some existing publications, and two kinds of methods proposed, i.e. market-based methods and direct control methods. Market-based methods include the day-ahead dynamic tariff, distribution capacity market, intra-day shadow price and flexibility service market [14]. For example, a congestion fee based market mechanism is proposed in [15] where the DSO predicts possible congestions for the coming trading day and publishes tariffs prior to the clearing of the day-ahead market to alleviate distribution congestions; the notion of flexibility clearing houses (FLECH) is proposed in [16] to enable small scale DERs to participate in flexibility service trading; a novel plan that establishes an individual charging plan for each EV to mitigate distribution system congestion while meeting the requirements of EV owners is developed in [17]; three potential strategies for congestion management are presented in [18]. The direct control methods include network reconfiguration, active power control and reactive power control. For instance, an optimal reconfigurationbased dynamic tariff (DT) method considering feeder reconfiguration in calculating DT is proposed for congestion management and line loss reduction in distribution networks with high penetration of EVs in [19].

Demand response (DR) programs can be employed to alleviate system congestions by motivating the interactions between power system dispatchers and power consumers $[20,21]$. A broad range of potential benefits on system operations and market efficiencies can be expected by reasonable implementations of DR programs [22]. In the long run, the construction investments of transmission and distribution facilities can also be reduced by implementing appropriate DR schemes. An energy consumption optimization model for a given customer in response to hourly electricity prices is formulated in [23]. The locational marginal price intervals under wind uncertainty are calculated in [24] without the need of Monte Carlo simulations. A distributed real-time DR algorithm to determine the interactions among multiple utility companies and users is proposed in [25].

However, there exist technical and economical difficulties for the DSO to directly dispatch numerous dispersed DR resources. A commonly adopted solution is to introduce aggregators that take responsibilities of integrating DR resources, actively participating in the electricity market and managing financial risks of power consumers at the same time [26, 27]. Nonetheless, the operation of aggregators is influenced by the price signals in the electricity market, and their concentrated consumptions during price valley hours may also result in congestions. To the best of our knowledge, the distribution congestion management problem considering the uncertainties of DERs' power outputs and electricity market prices has not been studied comprehensively so far.

Given this background, a bi-level optimization framework of distribution congestion management is developed in this paper, where the uncertainties of DG generation outputs and market prices are modeled through robust optimization techniques. The economic dispatch of the distribution system is carried out in the upper-level to attain the overall energy acquisition and load interruption strategy, and the nodal prices in the distribution system can be calculated to direct the behaviors of aggregators. Subsequently, the aggregators will optimize the detailed energy consumptions of customers within their control to maximize their economic profits based on the nodal prices. The optimized energy schedules in the lower level model will be returned to the upper level model for verification. Through iterations between these two levels, distribution congestions can be relieved through DR schemes.

The remainder of this paper is organized as follows. A brief introduction to the congestion management framework of smart distribution systems is described in Sect. 2. In Sect. 3, a deterministic bi-level linear programming model with interruptible loads (ILs) and flexible loads coordinated to tackle distribution system congestion is formulated. A robust optimization model is introduced to address uncertain factors in Sect. 4. The performance of the developed model is demonstrated by case studies in Sect. 5 . Finally, conclusions are drawn in Sect. 6.

\section{Congestion management in smart distribution systems}

\subsection{Structure of smart distribution system}

The structure of a smart distribution system is described in Fig. 1. The electric power consumed by end-users can be provided not only through the transmission system, but also by the DG suppliers. On the basis of advanced information 


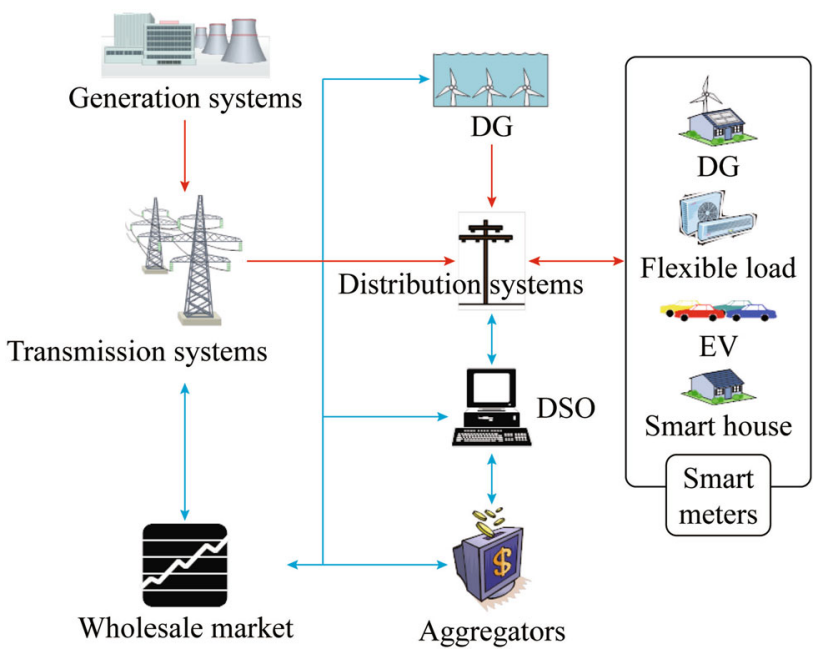

Fig. 1 Infrastructure of a smart distribution system

technology, distributed renewable generations such as wind turbines and solar panels are compatible with high permeability through coordination of energy storage devices and DR resources in the smart distribution system. For example, load levels of EVs and smart household appliances can be scheduled to accommodate more renewable generation output and meanwhile minimize the energy acquisition costs.

In fact, it is not realistic for numerous dispersed smallscale participants to directly participate in the wholesale electricity market. As an indispensable economic entity in the electricity market environment, aggregators play the roles of integrating demand side resources, participating in both electricity wholesale and retail markets, collecting data and carrying out statistical analysis, as well as communications with the DSO. By the optimal management of demand side resources, aggregators can gain profits, and improve the secure and economic operation of the distribution system.

The whole market structure of a smart distribution system is illustrated in Fig. 2 [28]. Numerous DGs, EVs and other demand side resources are managed by aggregators acting as emerging market entities in future distribution systems. With advanced information technology, the DSO who primarily takes responsibility for system security and

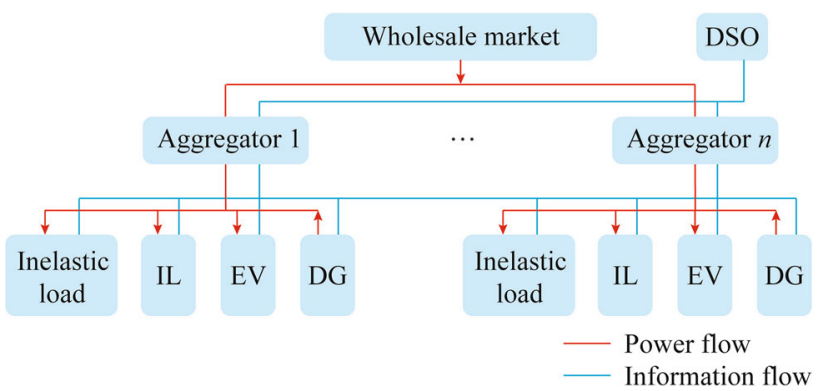

Fig. 2 Market structure of smart distribution system economics could interact with the aggregators for coordinated operation. Bidirectional flows of electric power and information exist simultaneously in smart distribution systems. As huge amount of data is expected by communications with and among numerous dispersed demand side resources, the data packing technique can be employed to handle the massive amount of data from smart meters at the load side.

\subsection{Process of congestion management}

All entities participating in congestion management can attain different degrees of benefit. The DSO minimizes the overall operation cost to meet all load demands in the system and maintains the load level within the allowed range to prevent the distribution facilities from being damaged. The aggregators charge some management fees to cover the operation and risk costs and make some profits for their services. The consumers attain professional assistants to construct their building energy management systems and be enabled to communicate with the aggregators, so that they can minimize their energy payment by cooperating with other consumers and interact with the DSO and power market operator without affecting their energy use.

Distribution system congestion can be alleviated by the comprehensive utilization of DR resources through nodal prices and other incentives. DR resources are assumed to be divided into flexible loads and ILs considering their various characteristics. Flexible loads such as EVs and smart household electric appliances could be transferred within a prescribed time period without interfering the electricity usage of consumers if assigned tasks can be completed. While for ILs such as industrial loads, lighting loads and others, economic compensation can be offered as incentives. In this paper, it is assumed that flexible loads are managed by aggregators, and compensation fees for ILs are provided to end-users by the DSO for implementing distribution system optimal scheduling.

Based on the above assumptions, the congestion management process of a smart distribution system can be implemented as follows:

1) The dispersed data of end-users including estimated electricity demands, flexible loads and ILs are collected from terminal smart meters by aggregators.

2) Massive amounts of data are sorted and merged by the data packing technique to reduce the problem scale by aggregators.

3) The processed data are submitted to the DSO.

4) DG power outputs, electricity wholesale market prices and their variable ranges are forecasted by the DSO.

5) Optimal solutions are attained by employing the presented optimization model. 
6) The aggregators are informed in advance of the load interruption schedule and nodal prices during the day.

7) The schedule of energy acquisition from the transmission system and load interruptions are carried out by the DSO, and flexible loads are properly managed through nodal prices by the aggregators.

\section{Deterministic bi-level programming model for congestion management}

\subsection{Upper-level model}

The congestion management framework developed in this work consists of two levels. In the upper-level model, the optimal power flow (OPF) of the distribution system during the day is conducted to attain the energy acquisition and load interruption schedule and calculate nodal prices in each trading period of the day. The DSO, the decisionmaker of the upper-level, aims at minimizing the total operation cost of the distribution system by appropriately scheduling energy acquisition and effectively utilizing ILs. DCOPF is used to attain the nodal prices of active power since the attained active power flow results by DC power flow are close to those obtained by AC power flow with acceptable errors but with much less computation time, although reactive power flow results and node voltage magnitudes suffer large errors with the DC formulations. Extensive simulation results of sample power systems exhibit good convergence of the DCOPF. Recently, a linearized OPF for active distribution system with reactive power flow and voltage constraints considered is proposed in [29] to mitigate the problem of the high $R / X$ ratio and incapability of addressing losses.

As the compensation fees for ILs are all supposed to be directly paid to consumers by the DSO and aggregators do not get any benefit from it, the quantities of load interruption during the day are globally optimized throughout the distribution system in the upper-level with the DSO as the decisionmaker, and meanwhile, flexible loads are properly managed with nodal prices by the aggregators in the lower-level.

Based on the above description, the decision variables of the upper-level are the power purchasing quantities from the transmission system and the loads interrupted in each period. The objective function of the upper-level could be formulated as (1) which consists of two components, namely the power purchasing costs from the wholesale market and the compensation costs for ILs.

The constraints of the upper-level model include: power balances constraints (2), distribution line capacity constraints (3), IL constraints (4), and constraints of power purchased from the transmission system (5).

$$
\min \sum_{t}^{T_{\text {sum }}} \sum_{i=1}^{N_{d}} \lambda_{t} P_{i n, i, t}+p_{I L, i, t} P_{I L, i, t}
$$

s.t.

$$
P_{i n, i, t}=\sum_{i=1}^{N_{d}}\left(P_{L, i, t}-P_{I L, i, t}+P_{a, i, t}+\sum_{e=1}^{N_{E V, i}} P_{e, i, t}-P_{D G, i, t}\right)
$$

$$
\begin{aligned}
& \mid \sum_{i=1}^{N_{d}} h_{m i}\left(P_{i n, i, t}+P_{D G, i, t}-P_{L, i, t}+P_{I L, i, t}\right. \\
& \left.\quad-\sum_{a=1}^{N_{S m a r t, i}} P_{a, i, t}-\sum_{e=1}^{N_{E V, i}} P_{e, i, t}\right) \mid \leq \bar{F}_{m} \\
& 0 \leq P_{I L, i, t} \leq \bar{P}_{I L, i} \\
& \underline{P}_{i n, i} \leq P_{i n, i, t} \leq \bar{P}_{i n, i}
\end{aligned}
$$

where $\lambda_{t}$ and $P_{i n, i, t}$ respectively denote the wholesale power market price and injected power of bus $i$ from the transmission system at time $t ; p_{I L, i, t}$ denotes the load interruption compensation price of bus $i$ at time $t ; P_{L, i, t}, P_{I L, i, t}, P_{a, i, t}, P_{e, i, t}$ and $P_{D G, i, t}$ respectively denote the inelastic loads, the ILs, the $a$-th group of smart household appliance loads, the $e$-th EV loads and the DG power injection of bus $i$ at time $t ; h_{m i}$ denotes the power transfer distribution factor of branch $m ; \bar{F}_{m}$ denotes the distribution line capacity of branch $m ; \bar{P}_{I L, i}$ denotes the upper limit of the ILs at bus $i ; \bar{P}_{i n, i}$ and $\underline{P}_{i n, i}$ denote the upper and lower limits of the power purchased from the transmission system at bus $i ; N_{d}$, $N_{S m a r t, i}$ and $N_{E V, i}$ respectively denote the number of buses in the distribution system, the number of smart household appliances and the number of EVs at bus $i ; T_{\text {sum }}$ is the number of time periods.

\subsection{Lower-level model}

As mentioned above, the aggregators would spontaneously schedule the flexible loads under control according to the nodal prices published by the DSO in the lower-level to minimize energy consumption costs. The nodal prices calculated in the upper-level include two parts, namely the wholesale market prices and the congestion fee which is additionally charged if congestion occurs. Hence, the nodal price can not only guide the flexible power consumption in periods with lower market prices, but also prevent the distribution system from congestion.

In the lower-level problem, the following assumptions are made in the decision-making process of each aggregator in the distribution system:

1) Electric power is supplied to end-users at fixed prices.

2) Each aggregator is assumed to be a price taker whose market power is sufficiently small to influence the market prices. 
3) A long-term contract is signed with DG suppliers to purchase renewable energy as much as possible at a fixed low price on the premise of ensuring the security of the power system.

4) Flexible loads can be categorized into several different groups according to their temporal availabilities and individual characteristics. For example, EVs could function as flexible loads if and only if they are physically connected to the distribution system concerned, and their available time periods rely on their daily mileages, battery characteristics and driving preferences. On the other hand, the smart household electric appliances managed by a smart house management system should accomplish some specified tasks during specified periods, e.g. air conditioning devices should maintain the indoor temperatures within certain intervals throughout the day. Thus, the flexibility constraints of their electricity consumptions may change over time, and are greatly influenced by external factors as well. As a result, these two types of flexible loads are modeled discriminately in this paper to embody their own characteristics.

Based on the above assumptions, the optimal consumption schedule of smart household appliances and EVs for each aggregator is developed. The decision variables of the lower-level model are the quantities of smart household appliance loads and EV charging loads consumed in each period. The objective of each aggregator is to maximize its profit, as formulated in (6). The profit of each aggregator can be represented by three parts: the revenues from electric power sale, the power purchasing costs from the wholesale market, the DG power purchasing costs.

The lower-level constraints include: the smart household appliance load constraints (7), the electric consumption quantity constraints of smart household appliances (8)(10), the EV load constraints (11), the EV's charging quantity constraints (12)-(14).

$$
\begin{aligned}
& \max \sum_{t}^{T_{s u m}} \sum_{i \in A_{n}}\left[\bar{p}\left(P_{L, i, t}-P_{I L, i, t}+P_{a, i, t}+\sum_{e=1}^{N_{E V, i}} P_{e, i, t}\right)\right. \\
& \quad-\lambda_{i, t}\left(P_{L, i, t}-P_{I L, i, t}+P_{a, i, t}+\sum_{e=1}^{N_{E V, i}} P_{e, i, t}-P_{D G, i, t}\right) \\
& \left.\quad-p_{D G} P_{D G, i, t}\right] \\
& \text { s.t. } 0 \leq P_{a, i, t} \leq \bar{P}_{a, i} \\
& \underline{Q}_{a, i, n} \leq \sum_{t=t_{a, s}}^{t_{a, n}} P_{a, i, t} \Delta t \leq \bar{Q}_{a, i, n} \\
& t_{a, s} \leq t_{a, n} \leq t_{a, f}
\end{aligned}
$$

$\sum_{t=t_{a, s}}^{t_{a, f}} P_{a, i, t} \Delta t=Q_{a, i, s u m}$

$\underline{P}_{E V} \leq P_{e, i, t} \leq \bar{P}_{E V}$

$\underline{S}_{E V} \leq \sum_{t=t_{e, s}}^{t_{e, n}} P_{e, i, t} \eta_{c} \Delta t \leq \bar{S}_{E V}$

$t_{e, s} \leq t_{e, n} \leq t_{e, f}$

$\sum_{t=t_{e, s}}^{t_{e, f}} P_{e, i, t} \eta_{c} \Delta t=Q_{E V, i}$

where $\bar{p}, \lambda_{i, t}$ and $p_{D G}$ respectively denote the fixed retail price to consumers, the nodal price of bus $i$ at time $t$, and the wholesale price of DG; $\bar{P}_{a, i}$ denotes the upper limit of the $a$-th smart household appliance load; $\bar{Q}_{a, i, n}$ and $\underline{Q}_{a, i, n}$ respectively denote the upper and lower limits of the electric consumption quantity of the $a$-th smart household appliance within a prescribed period of time, and $Q_{a, i, \text { sum }}$ denotes the power consumption for designated tasks; $t_{a, s}$ and $t_{a, f}$ respectively denote the starting and end time for the $a$-th smart household appliance load, and $t_{a, n}$ denotes a time period between them; $\bar{P}_{E V}$ and $\underline{P}_{E V}$ respectively denote the upper and lower limits of an individual EV load; $\bar{S}_{E V}$ and $\underline{S}_{E V}$ respectively denote the upper and lower limits of an individual EV's charging status; $\eta_{c}$ denotes the charging efficiency of EVs; $Q_{E V, i}$ denotes the total charging quantity of EVs at bus $i ; t_{e, s}$ and $t_{e, f}$ respectively denote the starting and end time for the $e$-th EV load, and $t_{e, n}$ denotes a time period between them.

\section{Robust bi-level programming model for congestion management}

\subsection{Modeling of uncertain quantities}

\subsubsection{Modeling uncertain renewable generation outputs}

With intermittent, volatile and uncertain attributes, numerous different types of renewable generations widely distributed at the load side have various output characteristics, and are difficult to be accurately modeled with probability. The DG power output of bus $i$ at time $t$ is assumed to vary in a certain range, which can be described as

$\tilde{P}_{D G, i, t} \in\left[\bar{P}_{D G, i, t}-\hat{P}_{D G, i, t}, \bar{P}_{D G, i, t}+\hat{P}_{D G, i, t}\right]$

where $\tilde{P}_{D G, i, t}, \bar{P}_{D G, i, t}$ and $\hat{P}_{D G, i, t}$ denote the uncertain DG power output, the forecasted DG power output, the maximum deviation of DG power output of bus $i$ at time $t$, respectively. 
The constrained variables $\Pi_{t}$ can be introduced to control the numbers of DGs with significant deviations of electric power outputs at time $t$, so that the conservatism of the proposed model can be regulated [30, 31]. For instance, $\Pi_{t}=0$ means that the power deviation at time $t$ is small; $\Pi_{t}=\left|J_{D G}\right| / 2$ means that at least half of DGs' outputs may deviate from the forecasted values significantly. Therefore, it can be seen that the larger the value of $\Pi_{t}$ is, the more conservative the solution could be. $\Pi_{t} \leq\left|J_{D G}\right|,\left|J_{D G}\right|$ is the number of buses with connected DGs.

Based on the above description, it can be assumed that in extreme scenarios, DG power outputs at a designated bus may reach the upper limit, the lower limit or the forecasted value, and the number of buses whose power outputs reach the limits will not be larger than $\Pi_{t}$. As a result, the uncertain set of DG power outputs can be represented as

$$
\begin{aligned}
\Pi & =\left\{\tilde{P}_{D G, i, t} \in \Re^{|I| \times|T|}: \tilde{P}_{D G, i, t}\right. \\
& \left.=\bar{P}_{D G, i, t}+x_{D G, i, t} \hat{P}_{D G, i, t}, \forall t \in T, \forall i \in I\right\}
\end{aligned}
$$

where

$-1 \leq x_{D G, i, t} \leq 1$

$-\Pi_{t} \leq \sum_{i \in J_{D G}} x_{D G, i, t} \leq \Pi_{t}$

where $x_{D G, i, t}$ represents the deviation degree of DG power outputs. If $x_{D G, i, t}=1 /-1 / 0$, then the DG power output of bus $i$ at time $t$ reaches the upper limit/the lower limit/the forecasted value. $T$ and $I$ respectively denote the set of time periods during the day and buses with connected DGs.

\subsubsection{Modeling uncertain electricity market prices}

Suppose that the day-ahead market price at time $t$ varies in a certain range, as described by

$\tilde{\lambda}_{t} \in\left[\bar{\lambda}_{t}-\hat{\lambda}_{t}, \bar{\lambda}_{t}+\hat{\lambda}_{t}\right]$

where $\tilde{\lambda}_{t}, \bar{\lambda}_{t}$ and $\hat{\lambda}_{t}$ denote the uncertain electricity market price, the predicted electricity market price and the maximum price deviation at time $t$, respectively.

To regulate the conservatism of the solution, a constrained variable $\Gamma$ is employed to control the total electricity price offset, $\Gamma \leq 24$. Conservatism of the solution changes as the value of $\Gamma$ varies. The smaller the value of $\Gamma$ is, the less uncertain the market prices will be. The uncertain set of day-ahead electricity market prices can be described as follows:

$\Gamma=\left\{\tilde{\lambda}_{t}=\bar{\lambda}_{t}+\Delta \lambda_{t}, \forall t \in T\right\}$

where

$-\hat{\lambda}_{t} \leq \Delta \lambda_{t} \leq \hat{\lambda}_{t}$
$-\Gamma \hat{\lambda}_{t} \leq \sum_{t \in T} \Delta \lambda_{t} \leq \Gamma \hat{\lambda}_{t}$

When $\Gamma=0$, the market price fluctuation in each period is small; When $\Gamma=8$, the market prices significantly deviate in at least 8 periods; $\Gamma=24$ represents extreme scenarios that market prices of all 24 periods may reach their limits.

\subsection{Robust optimization theory}

A robust approach to solve linear programming problems with uncertain data was first proposed in 1970s by Soyster [30]. With this approach, a suboptimal solution that is feasible for all data in a convex set can be attained so that the impact of data uncertainties on the quality and feasibility of solutions can be considered. However, optimality may be sacrificed in order to ensure the robustness. To address the issue of over-conservatism, an uncertain linear problem with ellipsoidal uncertainties is considered to attain a less conservative solution [31].

An initial programming problem with uncertain data is formulated as follows:

$\min _{x} \boldsymbol{c}^{T} \boldsymbol{x}$

s.t. $\quad \boldsymbol{A x} \leq \boldsymbol{b}$

$\boldsymbol{l} \leq \boldsymbol{x} \leq \boldsymbol{u}$

where $\boldsymbol{c} \in \mathbf{R}^{n}, \boldsymbol{x} \in \mathbf{R}^{n}, \boldsymbol{A} \in \mathbf{R}^{m \times n}, \boldsymbol{b} \in \mathbf{R}^{m}, \boldsymbol{l} \in \mathbf{R}^{n}, \boldsymbol{u} \in \mathbf{R}^{n}$.

In the above model, only matrix $\boldsymbol{A}$ includes uncertain data. Considering the $i$ th row of matrix $\boldsymbol{A}$, let $J_{i}$ represents the set of coefficients in row $i$ that are subject to uncertainty. Each entry $a_{i j}\left(j \in J_{i}\right)$ is modeled as a symmetric and bounded random variable that takes values in $\left[\bar{a}_{i j}-\hat{a}_{i j}, \bar{a}_{i j}\right.$ $+\hat{a}_{i j}$. The robust peer model can be formulated as

$\min _{x, y, p, q} \boldsymbol{c}^{T} \boldsymbol{x}$

s.t. $\quad \sum_{j=1}^{n} \bar{a}_{i j} x_{j}+\Gamma_{i} q_{i}+\sum_{j \in J_{i}} p_{i j} \leq b_{i}, \forall i$

$q_{i}+p_{i j} \geq \hat{a}_{i j} y_{j}, \forall i, j \in J_{i}$

$-y_{j} \leq x_{j} \leq y_{j}, \forall j$

$\boldsymbol{l} \leq \boldsymbol{x} \leq \boldsymbol{u}$

$p_{i j} \geq 0, \forall i, j \in J_{i}$

$y_{j} \geq 0, \forall j$

$q_{i} \geq 0, \forall i$

where an integer $\Gamma_{i}$ is introduced to restrict variation degrees of each inequality constraint; $\Gamma_{i}$ takes values in $[0$, $\left.\left|J_{i}\right|\right] ;\left|J_{i}\right|$ represents the element number in set $J_{i}$.

The parameter $\Gamma_{i}$ controls the trade-off between the probabilities of constraint violations. When $\Gamma_{i}=\left|J_{i}\right|$, a 
robust solution will be deterministically feasible as all possible values of uncertain coefficients being taken, even if more than $\left|J_{i}\right|$ changes, the robust solution will be feasible with a very high probability. The probability bounds of constraint violation can be calculated as within $\exp \left(-\Gamma_{i}^{2} / 2\left|J_{i}\right|\right)$, which can be utilized to evaluate the solution robustness when the probability distribution of random variables cannot be precisely described.

Considering uncertainties in the coefficients of the objective function, the model could be transformed into the following form

$\min z$

$c^{T} \boldsymbol{x} \leq z$

where $z$ is an auxiliary variable that transforms the model into forms of uncertain coefficients only existing in constraints.

\subsection{Robust bi-level programming model for congestion management}

The decision-making process of a bi-level programming problem can be classified into the decentralized and centralized modes. The decentralized mode is employed in this work. The definition of the robust solution of a bi-level programming problem with uncertain coefficients can be influenced by the dependency degree between the upper and lower levels in the decision-making process. In the decentralized decision-making mode, the uncertain factors in each level will not influence the decision-making process of the other one. So the robust peer model of each level can be derived independently. Based on the above, a robust peer model of the bi-level programming problem with uncertain values of DG power outputs and electricity market prices considered can be attained by utilizing the robust linear optimization theory.

Given the electricity market price $\tilde{\lambda}_{t} \in\left[\bar{\lambda}_{t}-\hat{\lambda}_{t}, \bar{\lambda}_{t}+\hat{\lambda}_{t}\right]$, a robust model derived from the upper-level can be formulated as follows:

$\min \sum_{t}^{T_{\text {sum }}} T_{t}$

s.t.

$-T_{t}+\Gamma w+\sum_{t \in T} v_{t}+\bar{\lambda}_{t} \sum_{i=1}^{N_{d}} P_{i n, i, t}+\sum_{i=1}^{N_{d}} \bar{p}_{I L, i, t} P_{I L, i, t} \leq 0$

$w+v_{t} \geq \hat{\lambda}_{t} y_{t}$

$$
-y_{t} \leq \sum_{i=1}^{N_{d}} P_{i n, i, t} \leq y_{t}
$$

$w \geq 0$

$v_{t} \geq 0$

$y_{t} \geq 0$

$P_{i n, i, t}=\sum_{i=1}^{N_{d}}\left(P_{L, i, t}-P_{I L, i, t}+P_{a, i, t}+\sum_{e=1}^{N_{E V, i}} P_{e, i, t}-P_{D G, i, t}\right)$

$\left|h_{m i}\left(P_{i n, i, t}+P_{D G, i, t}-P_{L, i, t}+P_{I L, i, t}-P_{a, i, t}-\sum_{e=1}^{N_{E V, i}} P_{e, i, t}\right)\right| \leq \bar{F}_{m}$

$0 \leq P_{I L, i, t} \leq \bar{P}_{I L, i}$

$\underline{P}_{i n, i} \leq P_{i n, i, t} \leq \bar{P}_{i n, i}$

where $\Gamma$ is a constrained variable used to limit the variation degree of electricity market prices and it is supposed to be no more than $24 ; w$ and $v_{t}$ are both dual variables; $T_{t}$ is an auxiliary variable. Other variables are defined before.

Given the DG power output $\tilde{P}_{D G, i, t} \in\left[\bar{P}_{D G, i, t}-\hat{P}_{D G, i, t}\right.$, $\left.\bar{P}_{D G, i, t}+\hat{P}_{D G, i, t}\right]$, a robust model can be derived from the lower-level as

$\min \sum_{i \in A_{n}} R_{i}$

s.t.

$$
\begin{aligned}
& -R_{i}+\sum_{t}^{T_{\text {sum }}}\left[\left(\lambda_{i, t}-\bar{p}\right)\left(P_{a, i, t}+\sum_{e=1}^{N_{E V, i}} P_{e, i, t}\right)\right. \\
& \left.+\left(\Pi_{t} f_{t}+\sum_{k \in J_{D G}} g_{k, t}\right)\right] \leq \sum_{t}^{T_{\text {sum }}}\left[\left(\bar{p}-\lambda_{i, t}\right)\left(P_{L, i, t}-P_{I L, i, t}\right)\right. \\
& \left.+\left(\lambda_{i, t}-p_{D G}\right) \bar{P}_{D G, i, t}\right]
\end{aligned}
$$

$f_{t}+g_{k, t} \geq \hat{P}_{D G, k, t}\left(\lambda_{k, t}-p_{D G}\right), \forall k \in J_{D G}$

$f_{t} \geq 0$

$g_{k, t} \geq 0, \forall k \in J_{D G}$

$0 \leq P_{a, i, t} \leq \bar{P}_{a, i}$

$\underline{Q}_{a, i, n} \leq \sum_{t=t_{a, s}}^{t_{a, n}} P_{a, i, t} \Delta t \leq \bar{Q}_{a, i, n}$

$t_{a, s} \leq t_{a, n} \leq t_{a, f}$

$\sum_{t=t_{a, s}}^{t_{a, f}} P_{a, i, t} \Delta t=Q_{a, i, s u m}$

$\underline{P}_{E V} \leq P_{e, i, t} \leq \bar{P}_{E V}$ 
$\underline{S}_{E V} \leq \sum_{t=t_{e, s}}^{t_{e, n}} P_{e, i, t} \eta_{c} \Delta t \leq \bar{S}_{E V}$

$t_{e, s} \leq t_{e, n} \leq t_{e, f}$

$\sum_{t=t_{e, s}}^{t_{e, f}} P_{e, i, t} \eta_{c} \Delta t=Q_{E V, i}$

where $\Pi_{t}$ is a constrained variable used to limit the variation degree of DG power outputs. $\Pi_{t} \leq\left|J_{D G}\right|$, and $\left|J_{D G}\right|$ represents the number of buses with DG injections, $f_{t}$ and $g_{k, t}$ are both dual variables, $R_{i}$ is an auxiliary variable. Other variables are defined before.

\subsection{Solving approach}

The mathematical model presented in Sect. 4.3 is a bilevel linear programming problem, and can be solved with a highly efficient commercial solver CPLEX 12.4 in MATLAB environment. The iteration of the two levels continues until the convergence criterion is reached. In this work, the convergence criterion is specified as: the variation of the system operation cost, namely the total cost of power purchasing and load interruption, between two adjacent iterations is less than a prespecified $\varepsilon$.

\section{Case studies and discussions}

A Danish $0.4 \mathrm{kV}$ distribution system from the Bornholm Island with topology described in [32] is employed to demonstrate the performance of the proposed model. There are 33 cables and 33 buses in this system, and 12 DGs are assumed to be connected to the $0.4 \mathrm{kV}$ buses of the system. Electric power from the transmission system is injected into the distribution system through a $10 / 0.4 \mathrm{kV}$ transformer. The buses of the above test system are assumed to be managed by 3 aggregators. The parameters of the smart household appliance loads, EV loads and inelastic loads of each aggregator are shown in Table 1 and Fig. 3, respectively. The total load demand of the ILs is assumed to be $1 / 9$ of the inelastic load power. The line capacities of this system and the predicting wholesale market prices throughout the day are listed in Tables 2 and 3, respectively.

The wholesale electricity market prices are assumed to be variable within 90\% 110\%; DG power outputs are supposed to be injected into buses from 10146 to 10157 with forecasted values of $200 \mathrm{~kW}$ and possible deviations of $\pm 20 \%$. The charging efficiency of an EV is set as 0.95 . The negotiated price for purchasing distributed renewable generation is supposed to be $0.35 \mathrm{DKK} / \mathrm{kWh}$; the retail price to consumers is fixed at $0.6 \mathrm{DKK} / \mathrm{kWh}$; the IL compensation price is $0.6 \mathrm{DKK} / \mathrm{kWh}$. The following three scenarios are considered:

1) The deterministic model with sufficient distribution line capacities;

2) The deterministic model with insufficient distribution line capacities;

3) The robust model with insufficient distribution line capacities.

\subsection{Deterministic model with sufficient distribution line capacities}

Doubling the given value of each distribution line capacity so that the system congestion would not happen, a

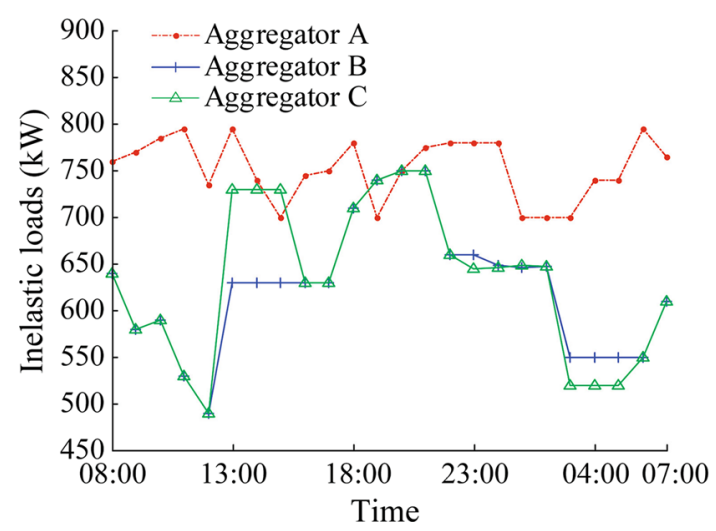

Fig. 3 Inelastic load parameters

Table 1 Parameters of smart household appliance loads and EV charging loads

\begin{tabular}{lcllllr}
\hline $\begin{array}{l}\text { Aggre- } \\
\text { gator }\end{array}$ & Bus number & $\begin{array}{l}\text { Time } \\
\text { period }\end{array}$ & $\begin{array}{l}\text { Smart household } \\
\text { appliance load }(\mathrm{kW})\end{array}$ & $\begin{array}{l}\text { Power consumption of smart } \\
\text { household appliance }(\mathrm{kWh})\end{array}$ & $\begin{array}{l}\text { EV's charging } \\
\text { load }(\mathrm{kW})\end{array}$ & $\begin{array}{l}\text { EV's charging } \\
\text { quantity }(\mathrm{kWh})\end{array}$ \\
\hline A & $528-538$, & $8: 00-19: 00$ & $0-150$ & 1200 & 0 & 0 \\
& $9922-9925$ & $20: 00-7: 00$ & $0-150$ & 1200 & $0-120$ & 910 \\
B & $359-360$, & $8: 00-19: 00$ & $0-160$ & 1280 & 0 & 0 \\
& 389,540 & $20: 00-7: 00$ & $0-160$ & 1280 & $0-130$ & 990 \\
C & $10146-10157$ & $8: 00-19: 00$ & $0-100$ & 800 & 0 & 0 \\
& & $20: 00-7: 00$ & $0-100$ & 800 & $0-120$ & 910 \\
\hline
\end{tabular}


deterministic solution can be attained with the proposed model in Sect. 3. A situation is first examined that the smart household appliances and EVs start to consume electric power at the starting point of their available time periods without the DR management scheme. The optimization results with and without DRs are compared in Table 4, and it is known that the total operation costs of the distribution system can be reduced by $14.15 \%$ with the coordinated utilization of DR resources.

The energy consumption schedules and nodal prices at bus 10155 throughout the day are depicted in Figs. 4 and 5, respectively. The nodal price of each bus is consistent with the wholesale electricity market price and no congestion fee is incurred. As can be observed, smart household appliance loads and EV charging loads, which can be freely managed if only given tasks are accomplished within a specified time duration, are automatically transferred to periods with lowest nodal prices since each distribution line capacity is sufficient. Moreover, if the wholesale electricity market price exceeds the IL price, the operation cost can be reduced by activating load interruptions.

Table 2 Line capacities of Danish $0.4 \mathrm{kV}$ distribution system

\begin{tabular}{ll}
\hline Line number & Line capacity (MW) \\
\hline 125LV-528, 125LV-533 & 20 \\
Others & 13 \\
\hline
\end{tabular}

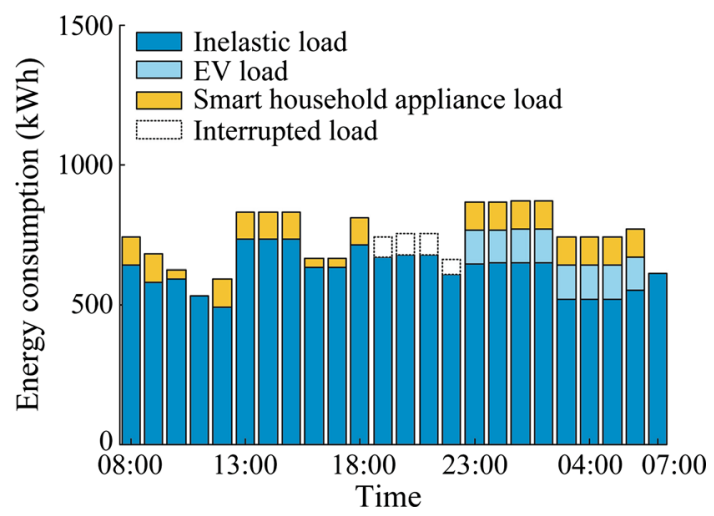

Fig. 4 Energy consumption schedule of bus 10155 in Scenario I

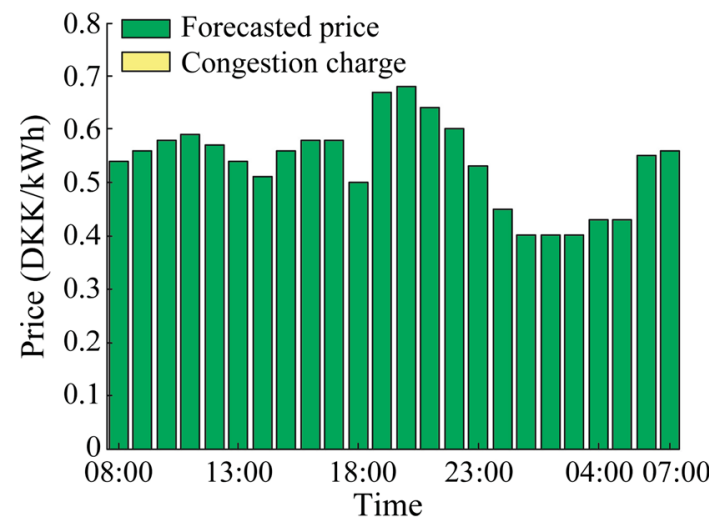

Fig. 5 Nodal price of bus 10155 in Scenario I

Table 3 Predicted wholesale market prices of a given day

\begin{tabular}{lcccccccc}
\hline Time & $8: 00$ & $9: 00$ & $10: 00$ & $11: 00$ & $12: 00$ & $13: 00$ & $14: 00$ & $15: 00$ \\
\hline Market price (DKK/kWh) & 0.54 & 0.56 & 0.58 & 0.59 & 0.57 & 0.54 & 0.51 & 0.56 \\
\hline Time & $16: 00$ & $17: 00$ & $18: 00$ & $19: 00$ & $20: 00$ & $21: 00$ & $22: 00$ & $23: 00$ \\
\hline Market price (DKK/kWh) & 0.58 & 0.58 & 0.50 & 0.67 & 0.68 & 0.64 & 0.60 & 0.53 \\
\hline Time & $24: 00$ & $1: 00$ & $2: 00$ & $3: 00$ & $4: 00$ & $5: 00$ & $6: 00$ & $7: 00$ \\
\hline Market price (DKK/kWh) & 0.45 & 0.40 & 0.40 & 0.40 & 0.43 & 0.43 & 0.55 & 0.56 \\
\hline
\end{tabular}

Table 4 Optimization results with and without DRs in Scenario I

\begin{tabular}{|c|c|c|c|c|c|c|}
\hline \multirow[t]{2}{*}{ Parameters } & \multicolumn{2}{|c|}{ Power purchase from wholesale market } & \multicolumn{2}{|c|}{ Load interruption } & \multicolumn{2}{|l|}{ Sum } \\
\hline & With DR & Without DR & With DR & Without DR & With DR & Without DR \\
\hline Energy quantity (MWh) & 525.876 & 610.463 & 7.516 & 0 & 533.392 & 610.463 \\
\hline Corresponding cost (DKK) & 276686.0 & 327530.0 & 4510.0 & 0 & 281196.0 & 327530.0 \\
\hline
\end{tabular}




\subsection{Deterministic model with insufficient distribution line capacities}

With the given parameters, the deterministic model presented in Sect. 3 is implemented to demonstrate the effectiveness of the proposed congestion management method. Comparisons of day-ahead schedules of Scenario I and Scenario II are shown in Table 5. Compared with Scenario I, it can be inferred that the overall operation cost of the distribution system increases when the distribution line capacities are insufficient. There are two reasons for this: 1) smart household appliance loads and EV charging loads are guided to periods with relatively higher market prices for alleviating the system congestion; 2) compensation incentives are provided to customers for participating in load interruption schemes.

The loading levels of the line from bus 528 to bus 529 with and without DRs are compared in Fig. 6. From Fig. 6, it can be found that the power flow through the distribution line 528-529 would exceed its upper limit during peak periods with concentrated energy consumption. The overload problem can be mitigated by flexible loads as guided by nodal prices and ILs as motivated by compensation fees.

The energy consumption schedule and nodal prices at bus 10155 in a given day are depicted in Figs. 7 and 8, respectively. By comparing Figs.7 and 8, it can be inferred that system congestion may be caused by intensive power consumption at 11:00 PM-02:00 AM when the nodal price is lower. Through the congestion charge, some smart household appliance loads and EV charging loads are transferred to other periods so that the system congestion is alleviated. However, the nodal prices maintain consistent with the electricity wholesale market prices and no congestion fee is incurred to the buses whose load levels have nothing to do with the line loading of line 528-529.

\subsection{Robust model with insufficient distribution line capacities}

The uncertain model of the congestion management problem presented in Sect. 4 is implemented to attain the robust solution that is immune to the uncertainties of DG power outputs and wholesale market prices in this

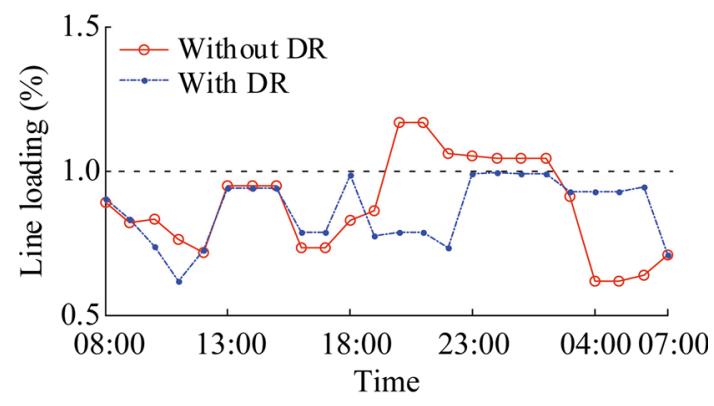

Fig. 6 Comparisons of line loading levels with and without DRs in Scenario II

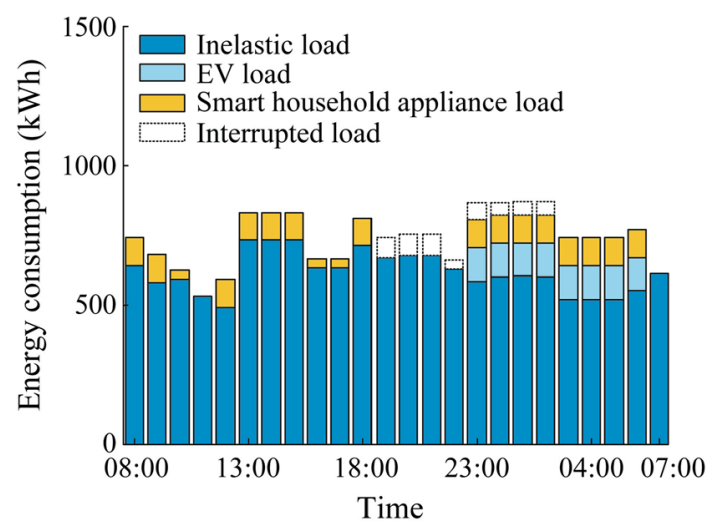

Fig. 7 Energy consumption schedule of bus 10155 in Scenario II

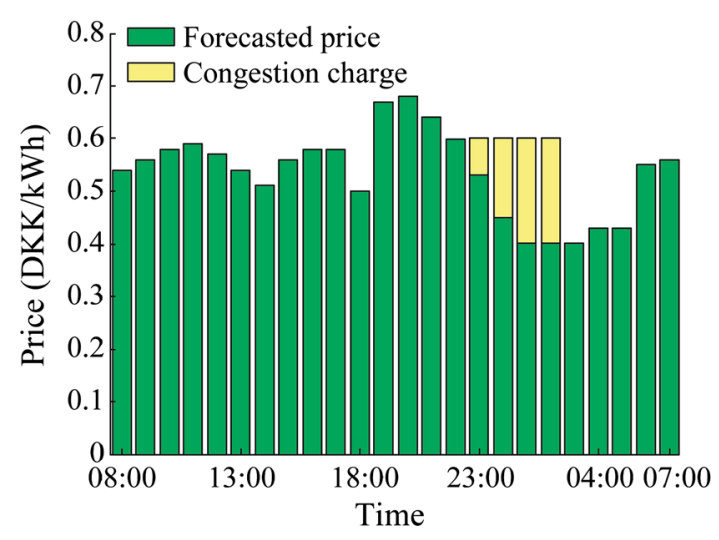

Fig. 8 Nodal prices of bus 10155 in Scenario II

Table 5 Comparisons of day-ahead schedules of Scenario I and Scenario II

\begin{tabular}{|c|c|c|c|c|c|c|}
\hline \multirow[t]{2}{*}{ Parameters } & \multicolumn{2}{|c|}{ Power purchase from wholesale electricity market } & \multicolumn{2}{|c|}{ Load interruption } & \multicolumn{2}{|l|}{ Sum } \\
\hline & I & II & I & II & I & II \\
\hline Energy quantity (MWh) & 525.876 & 525.193 & 7.516 & 8.197 & 533.392 & 533.4 \\
\hline Corresponding cost (DKK) & 276686.0 & 277386.8 & 4510.0 & 4918.7 & 281196.0 & 282305.5 \\
\hline
\end{tabular}


Table 6 Comparisons of various indexes under different robustness requirements

\begin{tabular}{lllll}
\hline Scenario & A & B & C & D \\
\hline Energy acquisition from day-head wholesale market (MWh) & 525.193 & 522.573 & 515.180 & 513.155 \\
Interrupted load quantity (MWh) & 8.198 & 10.767 & 17.928 & 19.875 \\
Power purchasing cost from wholesale market (DKK) & 277387 & 275859 & 271580 & 270537 \\
Load interruption cost in a day (DKK) & 4919 & 6461 & 10757 & 21.186 \\
\hline
\end{tabular}

section. Suppose that DG power outputs and wholesale market prices vary in their respective ranges, various robust solutions can be attained with constrained variables $\Gamma$ and

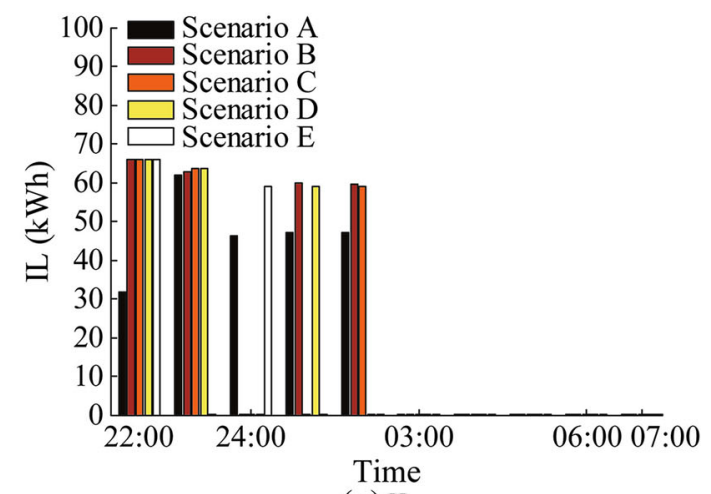

(a) ILs

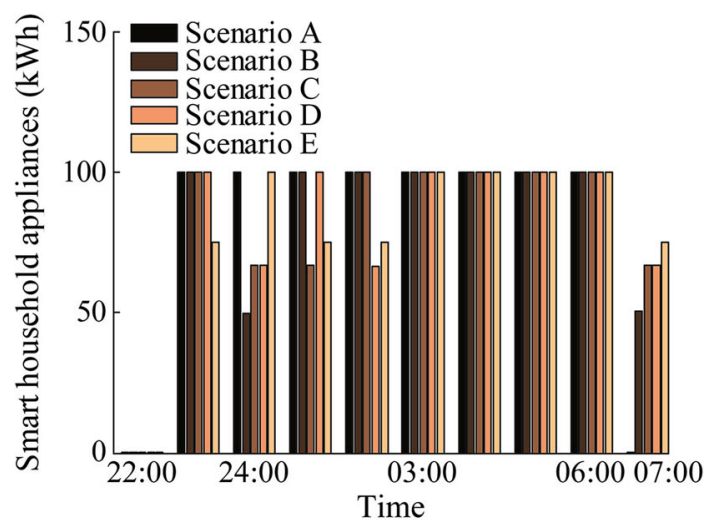

(b) Smart household appliances

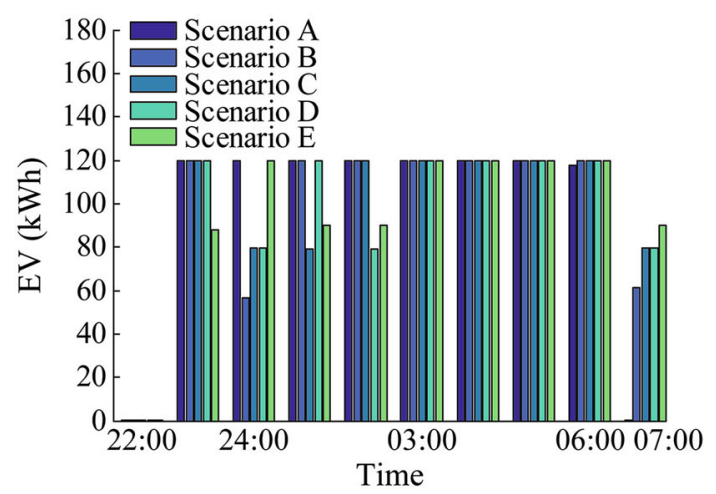

(c) EVs

Fig. 9 Comparisons of DRs at bus 10155 in different scenarios
$\Pi_{t}$ for regulating the trade-off between the optimality and robustness. The value of $\Gamma$ is taken in $[0,24]$, and the value of $\Pi_{t}$ in $[0,16], t=1,2, \ldots, 24$. In order to illustrate the performance of the developed model, solutions for 5 different scenarios are compared: A) $\left.\Gamma=0, \Pi_{t}=0 ; \mathrm{B}\right)$ $\Gamma=6, \Pi_{t}=4$; C) $\Gamma=12, \Pi_{t}=8$; D) $\Gamma=18, \Pi_{t}=12$; E) $\Gamma=24, \Pi_{\mathrm{t}}=16, t=1,2, \ldots, 24$. The results are listed in Table 6.

As shown in Table 6, from scenario A to scenario E, some economic efficiency is sacrificed for attaining more robustness in the decision-making process of the congestion management in the distribution system. When $\Gamma=0$ and $\Pi_{t}=0$, the most economical solution is achieved, but the uncertain factors are not taken into account; when $\Gamma=24$ and $\Pi_{t}=16$, the robust solution is sufficiently conservative to ensure the solution feasibility as all possible values of DG power outputs and wholesale market prices are within the respective ranges, or even if they are not in the ranges, the robust solution is still feasible with a very high probability. The trade-off between efficiency and robustness is well coordinated in scenarios B to D.

Comparisons of DRs from 10:00 PM to 7:00 AM and nodal prices throughout the day at bus 10155 in different scenarios are shown in Figs. 9 and 10, respectively. It can be seen that as the robust requirement changes, the ILs change accordingly and the nodal prices as well so as to guide the flexible loads to adjust their demands. Different economic and robustness requirements can be met by

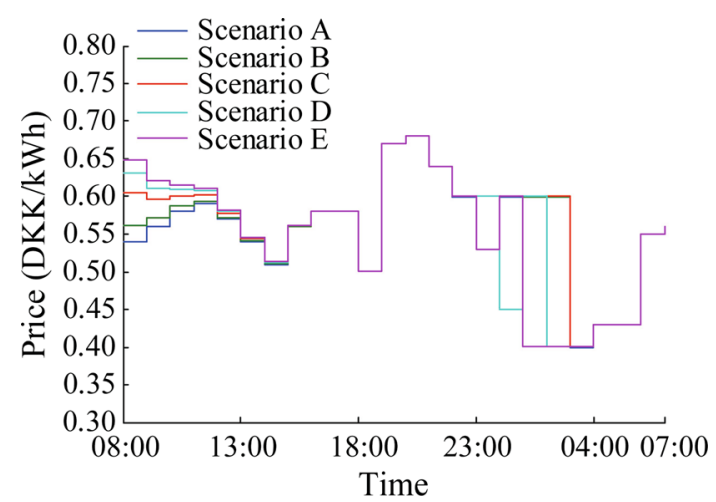

Fig. 10 Comparisons of nodal prices at bus 10155 under different scenarios 
implementing various congestion management strategies by the DSO.

In conclusion, negative impacts brought about by uncertainties can be alleviated by employing the robust linear programming approach. The trade-off between the economical efficiency and robustness of the optimization problem can be regulated according to various requirements. The characteristics of different kinds of DR resources, namely flexible loads and ILs are well utilized to alleviate the system congestion and minimize the total operation cost. A compromising solution which is able to balance the conservatism and optimality through coordinating various DR resources involved can be attained by the proposed robust congestion management model.

\section{Conclusion}

A robust congestion management model is developed for the DSO in this work. In the proposed model, the DR resources are coordinated to alleviate distribution system congestion. The economical efficiency and robustness of the congestion management strategies are balanced through robust optimization with uncertainties of DG power outputs and wholesale market prices taken into consideration. The structure and congestion management framework of a smart distribution system are first described. Subsequently, a robust bi-level programming model for the day-ahead congestion management in distribution systems is developed. Then, a highly efficient commercial solver CPLEX 12.4 is utilized in the MATLAB environment to solve the developed model. Finally, a Danish $0.4 \mathrm{kV}$ distribution system is employed to demonstrate the basic characteristics of the presented method.

Acknowledgements This work is jointly supported by National Basic Research Program of China (973 Program) (No. 2013CB228202), National Natural Science Foundsation of China (No. 51477151), Specialized Research Fund for the Doctoral Program of Higher Education (No. 20120101110112), and a Project by China Southern Power Grid Company (No. K-GD2014-192).

Open Access This article is distributed under the terms of the Creative Commons Attribution 4.0 International License (http:// creativecommons.org/licenses/by/4.0/), which permits unrestricted use, distribution, and reproduction in any medium, provided you give appropriate credit to the original author(s) and the source, provide a link to the Creative Commons license, and indicate if changes were made.

\section{References}

[1] Pantoja A, Quijano N (2011) A population dynamics approach for the dispatch of distributed generators. IEEE Trans Ind Electron 58(10):4559-4567
[2] Nick M, Cherkaoui R, Paolone M (2014) Optimal allocation of dispersed energy storage systems in active distribution networks for energy balance and grid support. IEEE Trans Power Syst 29(5):2300-2310

[3] Chen JC, Song XD (2015) Economics of energy storage technology in active distribution networks. J Mod Power Syst Clean Energy 3(4):583-588. doi:10.1007/s40565-015-0148-5

[4] Mathieu JL, Koch S, Callaway DS (2013) State estimation and control of electric loads to manage real-time energy imbalance. IEEE Trans Power Syst 28(1):430-440

[5] Smith R, Meng K, Dong ZY et al (2013) Demand response: a strategy to address residential air-conditioning peak load in Australia. J Mod Power Syst Clean Energy 3(1):223-230. doi:10.1007/s40565-013-0032-0

[6] Li CB, Tang SW, Cao YJ et al (2013) A new stepwise power tariff model and its application for residential consumers in regulated electricity markets. IEEE Trans Power Syst 28(1):300-308

[7] Zhong HW, Xie L, Xia Q (2013) Coupon incentive-based demand response: theory and case study. IEEE Trans Power Syst 28(2):1266-1276

[8] Madaeni SH, Sioshansi R (2013) Measuring the benefits of delayed price-responsive demand in reducing wind-uncertainty costs. IEEE Trans Power Syst 28(4):4118-4126

[9] Paull L, Li H, Chang LC (2010) A novel domestic electric water heater model for a multi-objective demand side management program. Electr Power Syst Res 80(12):1446-1451

[10] Yao WF, Zhao JH, Wen FS et al (2013) A hierarchical decomposition approach for coordinated dispatch of plug-in electric vehicles. IEEE Trans Power Syst 28(3):2768-2778

[11] Momber I, Morales-Espana G, Ramos A et al (2014) PEV storage in multi-bus scheduling problems. IEEE Trans Smart Grid 5(2):1079-1087

[12] Acha S, Green TC, Shah N (2011) Optimal charging strategies of electric vehicles in the UK power market. In: Proceedings of the 2011 IEEE PES innovative smart grid technologies conference, Anaheim, CA, USA, 13-16 Nov 2011, pp 1-8

[13] Sotkiewicz PM, Vignolo JM (2006) Nodal pricing for distribution networks: efficient pricing for efficiency enhancing DG. IEEE Trans Power Syst 21(2):1013-1014

[14] Huang S, Wu Q, Liu Z, et al (2015) Review of congestion management methods for distribution networks with high penetration of distributed energy resources. In: Proceedings of the 2015 IEEE PES innovative smart grid technologies conference, Istanbul, Turkey, 12-15 Oct 2015, pp 1-6

[15] Liu WJ, Wu QW, Wen FS et al (2014) Day-ahead congestion management in distribution systems through household demand response and distribution congestion prices. IEEE Trans Smart Grid 5(6):2739-2747

[16] Zhang CY, Ding Y, Nordentoft NC et al (2014) FLECH: a Danish market solution for DSO congestion management through DER flexibility services. J Mod Power Syst Clean Energy 2(2):126-133. doi:10.1007/s40565-014-0048-0

[17] Sundstrom O, Binding C (2012) Flexible charging optimization for electric vehicles considering distribution grid constraints. IEEE Trans Smart Grid 3(1):26-37

[18] Bach AP, Hu J, Heussen K (2012) Coordination strategies for distribution grid congestion management in a multi-actor, multiobjective setting. In: Proceedings of the 2012 3rd IEEE PES international conference and exhibition on innovative smart grid technologies, Berlin, Germany, 14-17 Oct 2012, pp 1-8

[19] Huang SJ, Wu QW, Cheng L et al (2016) Optimal reconfiguration-based dynamic tariff for congestion management and line loss reduction in distribution networks. IEEE Trans Smart Grid 7(3):1295-1303 
[20] Palensky P, Dietrich D (2011) Demand side management: demand response, intelligent energy systems, and smart loads. IEEE Trans Ind Inform 7(3):381-388

[21] Mancarella P, Chicco G (2013) Real-time demand response from energy shifting in distributed multi-generation. IEEE Trans Smart Grid 4(4):1928-1938

[22] Siano P (2014) Demand response and smart grids-a survey. Renew Sustain Energy Rev 30:461-478

[23] Conejo AJ, Morales JM, Baringo L (2010) Real-time demand response model. IEEE Trans Smart Grid 1(3):236-242

[24] Fang X, Wei YL, Li FX (2016) Evaluation of LMP intervals considering wind uncertainty. IEEE Trans Power Syst 31(3):2495-2496

[25] Deng RL, Yang ZY, Hou F et al (2015) Distributed real-time demand response in multiseller-multibuyer smart distribution grid. IEEE Trans Power Syst 30(5):2364-2374

[26] Shafiullah M, Al-Awami AT, Elamin IM (2015) Profit maximization planning of a load aggregator using electric vehicles through optimal scheduling of day ahead load. In: Proceedings of the 2015 international conference on intelligent system application to power systems, Porto, Portugal, 11-16 Sep 2015, pp 1-6

[27] Mathieu S, Ernst D, Louveaux Q (2013) An efficient algorithm for the provision of a day-ahead modulation service by a load aggregator. In: Proceedings of the 2013 innovative smart grid technologies Europe, Lyngby, Denmark, 6-9 Oct 2013, pp 1-5

[28] Arnold GW (2011) Challenges and opportunities in smart grid: a position article. Proc IEEE 99(6):922-927

[29] Yuan HY, Li FX, Wei YL et al (2016) Novel linearized power flow and linearized OPF models for active distribution networks with application in distribution LMP. IEEE Trans Smart Grid. doi:10.1109/TSG.2016.2594814

[30] Soyster AL (1973) Technical note-convex programming with set-inclusive constraints and applications to inexact linear programming. Oper Res 21(5):1154-1157

[31] Bertsimas D, Sim M (2004) The price of robustness. Oper Res 52(1):35-53

[32] Connell NO, Wu QW, Østergaard J (2012) Day-ahead tariffs for the alleviation of distribution grid congestion from electric vehicles. Electr Power Syst Res 92:106-114

Linna NI received the B.E. degree from Xi' an Jiaotong University, Xi' an, China, in 2008 and the M.E. degree from Huazhong University of Science and Technology, Wuhan, China, in 2011, both in electrical engineering. She is currently pursuing her Ph.D. degree in electrical engineering in the School of Electrical Engineering at Zhejiang University, China. Her research interests are demand response and Energy Internet.

Fushuan WEN received the B.E. and M.E. degrees from Tianjin University, Tianjin, China, in 1985 and 1988, respectively, and the
Ph.D. degree from Zhejiang University, Hangzhou, China, in 1991, all in electrical engineering. He joined the faculty of Zhejiang University in 1991, and has been a full professor and the director of the Institute of Power Economics and Information since 1997, and the director of Zhejiang University-Insigma Joint Research Center for Smart Grids since 2010 . He had been a university distinguished professor, the deputy dean of the School of Electrical Engineering and the director of the Institute of Power Economics and Electricity Markets in South China University of Technology, Guangzhou, China, from 2005 to 2009. Since May 2014, he has been a professor with Universiti Teknologi Brunei (Brunei University of Technology), Brunei, taking leaves from Zhejiang University. His research interests lie in power industry restructuring, power system alarm processing, fault diagnosis and restoration strategies, as well as smart grids and electric vehicles. Prof. Wen is an associate editor of IET Generation, Transmission \& Distribution, and an associate editor of ASCE's Journal of Energy Engineering.

Weijia LIU received the B.E. degree and Ph.D. degree from Zhejiang University, Hangzhou, China, in 2011 and 2016, respectively, both in electrical engineering. He was a project/research assistant with the Hong Kong Polytechnic University, the Technical University of Denmark and The University of Sydney, from August 2012 to February 2013, April 2013 to January 2014, and January 2015 to July 2015. His main research interests are power system restoration, smart grids and energy systems.

Jinling MENG received the B.E. degree and M.E. degree from Hunan University, Changsha, China, in 2009 and 2012, respectively, both in electrical engineering. He is an engineer with Electric Power Research Institute of Guangdong Power Grid Co., Ltd. His main research interests are smart grids and demand side management.

Guoying LIN received the B.E. degree from Zhejiang University, Hangzhou, China and M.E. degree from Shanghai Jiaotong University, Shanghai China, in 2005 and 2008, respectively, both in electrical engineering. $\mathrm{He}$ is a senior engineer with Electric Power Research Institute of Guangdong Power Grid Co., Ltd. His main research interests are smart grids and demand side management.

Sanlei DANG received the B.E. degree from Huazhong University of Science and Technology, Wuhan, China, and M.E. degree from South China University of Technology, Guangzhou, China, in 2006 and 2009, respectively, both in electrical engineering. He is a senior engineer with Electric Power Research Institute of Guangdong Power Grid Co., Ltd. His main research interests are smart grids and demand side management. 